\title{
Improved Generalized Method of Moment Estimators to Estimate Diffusion Models
}

\author{
Youngsoo $\mathrm{Choi}^{a} \cdot$ Yoon-Dong Lee ${ }^{b, 1}$ \\ ${ }^{a}$ Department of Mathematics, Hankuk University of Foreign Study \\ ${ }^{b}$ Sogang Business School, Sogang University
}

(Received August 4, 2013; Revised September 23, 2013; Accepted September 23, 2013)

\begin{abstract}
Generalized Method of Moment(GMM) is a popular estimation method to estimate model parameters in empirical financial studies. GMM is frequently applied to estimate diffusion models that are basic techniques of modern financial engineering. However, recent research showed that GMM had poor properties to estimate the parameters that pertain to the diffusion coefficient in diffusion models. This research corrects the weakness of GMM and suggests alternatives to improve the statistical properties of GMM estimators. In this study, a simulation method is adopted to compare estimation methods. Out of compared alternatives, NGMM-Y, a version of improved GMM that adopts the NLL idea of Shoji and Ozaki (1998), showed the best properties. Especially NGMM-Y estimator is superior to other versions of GMM estimators for the estimation of diffusion coefficient parameters.
\end{abstract}

Keywords: Generalized Method of Moment(GMM), Diffusion Models, New Local Linearization(NLL).

\section{1. 서론}

현대 금융공학의 기본적 요소인 이자율, 환율, 주가지수 등 금융자료들은 모두 시간적 변동성을 갖는다. 보통 이런 값들을 모형화하기 위하여는 확률편미분방정식의 형태로 표현되는 확산모형이 사용된다. 확 산모형의 경우에 있어서도, 모형모수의 추론을 위해서는 통계적 방법의 적용이 필요하다. 본 연구에서 는 확산모형에 대한 통계적 추론문제를 다루게 된다. 확산모형의 추론과 관련하여 다양한 연구들이 이 루어져 왔다. 확산모형의 추론에는 오일러근사법, 일반화적률추정법 $(\mathrm{GMM})$, 최대우도추정법, 베이즈 추정법 등 다양한 통계적추론법이 적용되어 왔다 (Beskos 등, 2006). GMM은 확산모형의 추정을 위하 여 매우 자주 사용되는 추정법임에도 불구하고 확산계수가 상수가 아니고 함수형태로 주어지는 확산모 형의 추정에서 모형모수를 정확하게 추정하지 못하는 문제점을 가지고 있는 것으로 알려지고 있다. 본 연구에서는 이와 같은 $\mathrm{GMM}$ 의 단점을 극복하기 위한 대안적 방법을 검토한다.

Research of the first author was supported by Hankuk University of Foreign Studies Research Fund of 2012. Research of the second author was supported by the Sogang University Research Grant of 2012 (201210023.01).

${ }^{1}$ Corresponding author: Associate Professor, Sogang Business School, Sogang University, 1 SinSoo-Dong,

Mapo-Gu, Seoul 121-742, Korea. E-mail: widylee@sogang.ac.kr 
확산모형은 미세입자의 움직임이나 금융자산의 가격변동을 모형화 할 때 자주 사용되는 수리적 모형이 다. 확산과정 $X_{t}$ 는, 추세계수 $\mu_{t}=\mu\left(\theta, t, X_{t}\right)$ 와 확산계수 $\sigma_{t}=\sigma\left(\theta, t, X_{t}\right)$ 를 갖는 다음과 같은 확률편 미분방정식의 해로서 주어지는 연속시간 마코프 확률과정을 말한다.

$$
d X_{t}=\mu_{t} d t+\sigma_{t} d W_{t} .
$$

확률편미분방정식 (1.1)의 해의 존재성에 대한 논의는 Kloeden과 Platen (1999, p.36)에서 찾을 수 있 다. 추세계수 $\mu_{t}$ 와 확산계수 $\sigma_{t}$ 의 형태에 따라 다양한 확산과정이 정의된다. 특히 추세계수와 확산 계수가 $\mu_{t}=\mu\left(\theta, X_{t}\right)$ 이고 $\sigma_{t}=\sigma\left(\theta, X_{t}\right)$ 인 형태로, 시간변수 $t$ 에 대하여 직접 영향을 받지 않으면 서 립쉬츠 연속성 조건(Lipschitz continuity condition)을 만족하는 경우를 이또확산과정(Itô diffusion process)이라 한다 (Oksendal, 2003, p.114, p.149). 본 연구에서는 논의의 단순화를 위하여 이또확산 과정을 중심으로 서술한다.

최근 확산모형에 대한 연구에서는 금융자산 가격의 특이한 변동 현상을 설명해야 하는 현실적 필요에 의하여 보다 다양한 형태의 확산모형이 제안되고 연구되고 있다. 대표적인 확산모형으로는 OrnsteinUhlenbeck(OU) 확률과정에 해당하는 Vasicek (1977) 모형, Cox 등 (1985)에 의해 제안된 CIR 모형, Cox (1975)가 제안한 Constant Elasticity of Variance 모형(CEV 모형) 등이 있다. 이자율 기간구조 연구에서 자주 사용되는, CKLS 모형이라 불리는, 모형 Chan 등 (1992) 모형은 선형적인 추세계수를 갖는 $\mathrm{CEV}$ 의 모형의 일종으로 다음 확률편미분방정식의 형태로 표현된다.

$$
d X_{t}=\alpha\left(\beta-X_{t}\right) d t+\sigma X_{t}^{\gamma} d W_{t} .
$$

$\mathrm{OU}$ 모형은 $\gamma=0$ 인 경우의 $\mathrm{CKLS}$ 모형에 해당하고, CIR 모형은 $\gamma=1 / 2$ 인 경우이다. 또 기하브 라운운동(Geometric Brownian Motion; GBM)은 Black-Scholes 옵션가격산정 모형에 사용되어 매우 잘 알려진 확산모형으로, 위 식 (1.2)에서 $\beta=0$ 이고 $\gamma=1$ 인 경우에 해당한다. 이 외에도 다양한 확 산모형이 금융현상의 모형화에 자주 고려되고 있고, Chan 등 (1992)에는 이에 대하여 잘 정리되어 있 다. 추세계수 $\mu_{t}$ 와 확산계수 $\sigma_{t}$ 를 정의할 때 특정 모수들을 포함하도록 하는 형태로 정의하는 방법이 자주 사용된다. 예를 들어 CKLS 모형은 모수 $\theta=(\alpha, \beta, \sigma, \gamma)$ 를 포함하게 되고, Vasicek 모형은 모수 $\theta=(\alpha, \beta, \sigma)$ 를 포함하게 된다. 이와 같이 추세계수와 확산계수에 모형모수가 개입되어 있음을 명시적 으로 나타내기 위하여 $\mu(\theta, \cdot)$ 혹은 $\sigma(\theta, \cdot)$ 와 같이 표현한다.

확산모형 $X_{t}$ 를 정의하기 위하여 사용하는 확률편미분방정식 (1.1)은 미세입자의 움직임이나 금융자산 의 가격변화에 대하여 매우 짧은 시간사이의 동적관계를 규정하는 모형이다. 이에 반하여 실제에서 확 산과정을 관측하여 얻게 되는 자료는 상당한 시간간격을 두고 관측되게 된다. 즉, 확산과정을 관측하여 얻은 값을 $\left\{X_{t_{i}}\right\}_{i=0}^{n}$ 이라 하면 관측시점 $t_{i}$ 들은 이산적인 특성을 갖는다. 규칙적인 시간간격으로 관측이 이루어진 경우를 가정하면 $\Delta=\left(t_{i+1}-t_{i}\right)$ 이다 $(\Delta>0)$. 결국 확산모형의 확률적 특성을 정의하는 확 률편미분방정식은 연속시간 모형인데 반하여, 관측되는 자료는 이산적인 시간간격으로 관측되는 것이므 로, 관측시점 사이의 모든 값들이 결측치가 되는 상황이다. 이런 이유로 확산모형의 추정을 위하여는 우 도추정법(likelihood estimation method)이나 베이즈추정법(Bayesian estimation method)과 같이 정 확한 우도함수를 알아야하는 추정방법을 적용하는 데는 어려움이 따른다.

근래에 들어 이런 어려움을 해결하여 확산모형에 대하여 우도추정법이나 베이즈추정법을 적용하려는 시 험적 연구가 이루어지고 있다 (Pederson, 1995; Durham와 Gallant, 2001; Elerian 등, 2001; Eraker, 2001; Aït-Sahalia, 2002). 이런 연구들에도 불구하고 확산모형의 추정을 위하여 우도추정법이나 베이 즈추정법을 적용하기 위한 방법들은 전체적으로 이론이 난해하고, 이를 이용하여 실제 추정을 하고자 하 는 경우에도, 적용 모형의 범용성이 결여되어 있거나, 소프트웨어로 구현이 어렵거나, 그 결과의 정확성 을 신뢰하기 어려운 경우들도 발생하는 등 실용성에 대한 의문이 제기되어 왔다. 
이런 이유로 확산모형의 추정을 위하여는 오일러근사법(Euler approximation scheme)이나 일반화적률 법(Generalized Method of Moment; GMM)과 같은 확산모형에 특화되어 개발된 방법들 혹은 다양 한 통계적모형에 대한 적용편의성이 높은 특이방법들이 주로 사용되어 왔다. Hurn 등 (2007)은 확산 모형에 대한 다양한 추정법들에 대하여 잘 정리하여 설명하고 있고, 각 추정법들의 성능을 비교하는 실 험 결과를 제시하고 있다. Hurn 등 (2007)이 제시한 모의실험 결과에 의하면, OU 모형이나 CIR 모형 을 기준으로 시험된 경우들에 대하여 오일러근사법이나 Shoji와 Ozaki (1998)가 제안한 국소선형근사 법(New Local Linearization; NLL)과 같이 비교적 단순한 형태의 근사법이 예상과 달리 통계적으로 정 확성이 상당히 높은 추정결과를 보여주고 있다. 또한 이들 방법은 그 원리가 단순한 이유로 구현이 쉽고 계산 속도 또한 다른 방법들에 비하여 빠르다는 장점을 보여주고 있다.

확산모형의 추정을 위하여 가장 자주 사용되는 방법은 Hansen (1982)에 의하여 제안된 GMM인 것으 로 알려지고 있다. GMM은 모형에서 얻어지는 모형적률에 관한 식과 표본에서 얻어지는 표본적률에 관한 식의 차이가 최소가 되도록 하는 모수값을 추정량으로 결정하는 방법으로, 직관적으로 이해가 쉽 고, 실제 응용이 용이하며, 모형의 복잡성에 영향을 덜 받는 이유로 인해서 널리 사용되는 추정방법이 다. Chan 등 (1992)은 확산모형인 CKLS 모형의 추정에 있어서 GMM을 적용하는 경우를 예로 들어 설명하고 있다. 반면 일반적으로 $\mathrm{GMM}$ 추정량이 갖는 통계적 성질은 최대우도추정량과 같은 다른 추 정량들에 비하여 상대적으로 열등하다는 것이 알려져 있다. Kim과 Lee (2011)은 CKLS 모형에 대하여 $\mathrm{GMM}$ 을 적용하는 경우 확산계수에 개입되는 모수 $\sigma, \gamma$ 에 대한 추정량의 성질이 다른 추정법들에 비하 여 매우 좋지 못함을 모의실험을 통해서 보이고 있다.

본 연구에서는 이런 인식하에서, NLL 추정법의 핵심적 요소를 GMM에 이식하여 GMM 추정량의 성질 을 개선하는 방법을 살펴보게 된다. NLL 방법의 핵심적 아이디어는 임의의 확산모형을, 그 정확한 우 도(likelihood) 함수가 알려져 있는 OU 모형으로 근사하여 근사적인 방법으로 최대우도추정법을 적용 하는 것이다. 다음 절에서는 GMM과 NLL 추정법에 대하여 살펴보고, 제 3 절에서는 $\mathrm{NLL}$ 추정법의 특 징을 $\mathrm{GMM}$ 에 이식하는 방법을 제안하게 된다. 제4절에서는 모의실험을 통하여 $\mathrm{CKLS}$ 모형을 중심으 로하여 NLL 근사법에 근거한 GMM(NLL-based GMM; 이하 NGMM 이라 함)을 다른 추정방법들과 비교하게 된다.

\section{GMM}

이 절에서는 확산과정 $X_{t}$ 에 대하여 매 $\Delta$ 시간 간격으로 관측하여 얻은 관측치 $x_{t+i \Delta}, i=0,1, \ldots$ 가 있 을 때 이로부터 $\mathrm{GMM}$ 추정량과 $\mathrm{NLL}$ 추정량을 구하는 방법을 살펴보게 된다. 간략한 표현을 위하여 $\mathrm{x}_{i}=x_{t+i \Delta}$ 라 하고, $\mathbf{x}=\left\{\mathrm{x}_{0}, \mathrm{x}_{1}, \ldots, \mathrm{x}_{n}\right\}$ 라 하자. Chan 등 (1992)은 식 (1.2)의 형태로 정의되는 CKLS 모형의 모수 $\theta=(\alpha, \beta, \sigma, \gamma)$ 를 추정하는 경우에 $\Delta=1$ 이라고 보고 GMM을 적용하는 추정방법에 대하 여 살펴보았다. 이를 보다 일반화하여 일반적인 확산모형 (1.1)의 경우와 일반적인 관측시간 간격 $\Delta$ 를 상정하고 일반화하여 $\mathrm{GMM}$ 을 적용하는 절차를 살펴보면 다음과 같다.

먼저 오일러근사법에 따라, $i=1,2, \ldots$ 에 대하여 다음과 같은 관계를 고려하자.

$$
\mathrm{X}_{i}-\mathrm{x}_{i-1} \sim \mathcal{N}\left(\mu_{i-1} \Delta, \sigma_{i-1}^{2} \Delta\right)
$$

여기서 $\mu_{i-1}=\mu\left(\theta, x_{i-1}\right)$ 이고 $\sigma_{i-1}=\sigma\left(\theta, x_{i-1}\right)$ 이다. 이때

$$
\begin{aligned}
\varepsilon_{i} & =\left(\mathrm{x}_{i}-\mathrm{x}_{i-1}\right)-\mu_{i-1} \Delta, \\
v_{i}^{2} & =\left\{\sigma\left(\mathrm{x}_{i-1}\right)\right\}^{2} \Delta
\end{aligned}
$$


라 하고, 크기가 $n \times J$ 인 도구변수행렬 $Z$ 의 열들은 도구변수 $\mathbf{z}_{j}=\left(z_{1, j}, z_{2, j}, \ldots, z_{n, j}\right)^{t}, j=1,2, \ldots, J$ 로 이루어져 있다. 즉 $Z=\left(\mathrm{z}_{1}, \mathrm{z}_{2}, \ldots, \mathrm{z}_{J}\right)$ 이다. 또

$$
\begin{aligned}
& \psi_{j}(Z ; \theta)=\frac{1}{n} \sum_{i=1}^{n} z_{i, j} \varepsilon_{i}, \quad j=1,2, \ldots, J_{1}, \\
& \psi_{j}(Z ; \theta)=\frac{1}{n} \sum_{i=1}^{n} z_{i, j}\left(\varepsilon_{i}^{2}-v_{i}^{2}\right), \quad j=\left(J_{1}+1\right), \ldots, J
\end{aligned}
$$

라 하자. 여기서 $J_{1}$ 은 $\varepsilon_{i}$ 와 관련된 $\psi_{i}$ 의 개수이고, $J_{2}$ 는 $\varepsilon_{i}^{2}$ 관련된 $\psi_{i}$ 의 개수이다. 또 $J=J_{1}+J_{2}$ 이 다. 혼동의 염려가 없는 경우 $\psi_{j}(Z ; \theta)$ 를 간략하게 $\psi_{j}(\theta), \psi_{j}(Z)$, 혹은 $\psi_{j}$ 로 나타내기로 하자. 이때 $\boldsymbol{\psi}_{J}=\left(\psi_{1}, \psi_{2}, \ldots, \psi_{J}\right)^{t}$ 라 하고, 표본의 크기 $n$ 을 함께 나타낼 필요가 있을 때는 $\boldsymbol{\psi}_{n, J}$ 로 나타내기로 하 자. 크기가 $J \times J$ 인 어떤 대칭 가중행렬 $\mathcal{W}$ 에 대하여

$$
Q_{n}(\theta)=\boldsymbol{\psi}_{J}^{t} \mathcal{W} \boldsymbol{\psi}_{J}
$$

를 최소화하는 $\theta$ 를 $\mathrm{GMM}$ 추정량으로 한다. 어떤 함수 $f(\theta)$ 를 $\theta \in \mathbb{R}^{p}$ 에 대하여 미분한 함수를 $\dot{f}(\theta)$ 로 나타내기로 하자. 즉 $\dot{\boldsymbol{\psi}}_{J}=(\partial / \partial \theta) \boldsymbol{\psi}_{J}$ 이다. 이때 도구변수 $\mathbf{z}_{j}$ 들이 모수 $\theta$ 와 무관한 경우라면,

$$
\begin{aligned}
& \dot{\psi}_{j}=-\frac{\Delta}{n} \sum_{i=1}^{n} z_{i, j} \dot{\mu}\left(\theta, \mathrm{x}_{i-1}\right), \quad j=1,2, \ldots, J_{1}, \\
& \dot{\psi}_{j}=-\frac{2 \Delta}{n} \sum_{i=1}^{n} z_{i, j}\left(\varepsilon_{i} \dot{\mu}\left(\theta, \mathrm{x}_{i-1}\right)+\sigma\left(\mathrm{x}_{i-1}\right) \dot{\sigma}\left(\mathrm{x}_{i-1}\right)\right), \quad j=\left(J_{1}+1\right), \ldots, J
\end{aligned}
$$

이고 $\dot{\boldsymbol{\psi}}_{J}=\left(\dot{\psi}_{1}^{t}, \dot{\psi}_{2}^{t}, \ldots, \dot{\psi}_{J}^{t}\right)^{t}$ 이다.

모수 $\theta$ 에 대한 $\mathrm{GMM}$ 추정량 $\hat{\theta}_{G M M}$ 은 적당히 설정된 정규조건(regularity condition) 하에서

$$
\left(\dot{\boldsymbol{\psi}}_{J}\left(\hat{\theta}_{G M M}\right)\right)^{t} \mathcal{W} \boldsymbol{\psi}_{J}\left(\hat{\theta}_{G M M}\right)=0
$$

을 만족하고, $n$ 이 커짐에 따라

$$
\sqrt{n} \boldsymbol{\psi}_{n, J} \stackrel{d}{\longrightarrow} \mathcal{N}_{J}(\mathbf{m}, V)
$$

인 성질이 만족되면,

$$
\sqrt{n}\left(\hat{\theta}_{G M M}-\theta_{0}\right) \stackrel{d}{\longrightarrow} \mathcal{N}_{p}\left(\mathbf{m}_{G M M}, V_{G M M}\right)
$$

이다. 여기서 $\theta_{0}$ 는 $\theta$ 의 참값이다. 또 $\dot{\boldsymbol{\psi}}_{0}=\dot{\boldsymbol{\psi}}_{J}\left(\theta_{0}\right)$ 라 하면,

$$
\begin{aligned}
\mathbf{m}_{G M M} & =-\left(\dot{\boldsymbol{\psi}}_{0}^{t} \mathcal{W} \dot{\boldsymbol{\psi}}_{0}\right)^{-1} \dot{\boldsymbol{\psi}}_{0}^{t} \mathcal{W} \mathbf{m}, \\
V_{G M M} & =\left(\dot{\boldsymbol{\psi}}_{0}^{t} \mathcal{W} \dot{\boldsymbol{\psi}}_{0}\right)^{-1} \dot{\boldsymbol{\psi}}_{0}^{t} \mathcal{W} V \mathcal{W} \dot{\boldsymbol{\psi}}_{0}\left(\dot{\boldsymbol{\psi}}_{0}^{t} \mathcal{W} \dot{\boldsymbol{\psi}}_{0}\right)^{-1}
\end{aligned}
$$

이고, 만약 $\mathcal{W}=V^{-1}$ 이라 하면 $V_{G M M}=\left(\dot{\boldsymbol{\psi}}_{0}^{t} V^{-1} \dot{\boldsymbol{\psi}}_{0}\right)^{-1}$ 이고, 이 경우가 $V_{G M M}$ 가 가장 작아지는 경우 이고 $\mathbf{m}=\mathbf{0}$ 라 할 때, $\hat{\theta}_{G M M}$ 이 최적이 되는 경우임이 널리 알려져 있다. 이 때 행렬 $V_{G M M}$ 의 크기에 관한 언급의 정확한 의미는 Heyde (1997)에 정의되어 있다. 
추정량 $\hat{\theta}_{G M M}$ 이 일치성을 갖는다고 가정하더라도, 즉 $n$ 이 커짐에 따라 $\hat{\theta}_{G M M} \stackrel{p}{\rightarrow} \theta_{0}$ 이라고 하더라도, $E\left[\sqrt{n}\left(\hat{\theta}_{G M M}-\theta_{0}\right)\right] \rightarrow 0$ 이 아닌 경우들을 상정할 수 있으므로, $\mathbf{m}=\mathbf{0}$ 이라는 가정을 하지 않는 경우 $\mathrm{GMM}$ 추정량의 평균제곱오차(Mean Squared Error; MSE)는

$$
\mathrm{MSE}=\frac{1}{n} \cdot\left(\dot{\boldsymbol{\psi}}_{0}^{t} \mathcal{W} \dot{\boldsymbol{\psi}}_{0}\right)^{-1} \dot{\boldsymbol{\psi}}_{0}^{t} \mathcal{W}\left(\mathbf{m m}^{t}+V\right) \mathcal{W} \dot{\boldsymbol{\psi}}_{0}\left(\dot{\boldsymbol{\psi}}_{0}^{t} \mathcal{W} \dot{\boldsymbol{\psi}}_{0}\right)^{-1}
$$

이고, 만약 $\mathcal{W}=\left(\mathrm{mm}^{t}+V\right)^{-1}$ 이라 하면

$$
\mathrm{MSE}=\frac{1}{n} \cdot\left[\dot{\boldsymbol{\psi}}_{0}^{t}\left(\mathbf{m m}^{t}+V\right)^{-1} \dot{\psi}_{0}\right]^{-1}
$$

이다.

여기서 도구변수(instrumental variable)의 가장 단순한 예로 $z_{i, j}=\left(\mathrm{x}_{i-1}\right)^{j-1}, i=1,2, \ldots, n, j=$ $1,2, \ldots, J$ 인 경우를 고려할 수 있다. 도구변수행렬 $Z$ 와 가중행렬 $W$ 의 선택 문제에 대하여는 뒷 절에 서 별도로 다루기로 한다.

Chan 등 (1992)은 이 때 $E\left[\varepsilon_{i}\right]=0$ 이고 $E\left[\varepsilon_{i}^{2}\right]=\left\{\sigma\left(\theta, x_{i-1}\right)\right\}^{2} \Delta$ 임을 가정하였다. 이 가정은 확산모형 (1.1)에 대한 오일러근사법 $X_{t+\Delta}-X_{t}=\mu\left(X_{t}\right) \Delta+\sigma\left(X_{t}\right) \cdot\left(W_{t+\Delta}-W_{t}\right)$ 이 정확하다고 가정할 때 얻 어지는 조건이다. 오일러근사법이 정확하다고 가정하면 $\mathbf{m}=0$ 이고, 크기가 $J \times J$ 인 분산공분산행렬 $V=\left\{v_{j, k}\right\}$ 는 $v_{j, k}=\lim _{n \rightarrow \infty} n E\left[\psi_{j} \psi_{k}\right]$ 이다. 여기서 $j, k=1,2, \ldots$ 에 대하여

$$
\begin{aligned}
& n E\left[\psi_{j} \psi_{k}\right]=\frac{1}{n} \sum_{i=1}^{n} z_{i, j} z_{i, k} v_{i}^{2}, \quad j, k=1,2, \ldots, J_{1}, \\
& n E\left[\psi_{j} \psi_{k}\right]=\frac{2}{n} \sum_{i=1}^{n} z_{i, j} z_{i, k} v_{i}^{4}, \quad j, k=\left(J_{1}+1\right), \ldots, J
\end{aligned}
$$

이고, $n E\left[\psi_{j} \psi_{k}\right]=0, j=1,2, \ldots, J_{1}, k=\left(J_{1}+1\right), \ldots, J$ 이다.

Chan 등 (1992)에서 이용된 오일러근사법에 근거한 $E\left[\varepsilon_{i}\right]=0$ 이고 $E\left[\varepsilon_{i}^{2}\right]=\left\{\sigma\left(\theta, x_{i-1}\right)\right\}^{2} \Delta$ 인 조건 은 확산모형 (1.1)에 대하여 일반적으로 성립하는 조건이 아니다. 일반적으로 $\varepsilon_{i}$ 가 갖는 분포적 특성 은, 오일러근사법 대신에, $X_{t}=x_{0}$ 인 조건이 주어진 경우 $X_{t+\Delta}$ 의 분포를 설명하는 전이확률밀도함수 $p^{\Delta}\left(x_{0}, x\right)$ 를 살펴봄으로써 보다 정확하게 알 수 있다. 다음에서는 기본적인 전이확률밀도의 개념과 이 를 근사하는 방법으로써의 NLL의 아이디어를 살펴보고 이를 이용하여 GMM을 개선하기 위한 대안으 로 $\mathrm{NGMM}$ 을 제시하게 된다. GMM 중 오일러근사법에 따라 식 (2.1)을 가정하여 GMM을 적용하는 경우를 NGMM과 구별하여 EGMM이라 부르기로 한다.

\section{NGMM: NLL에 근거한 GMM}

확산모형 (1.1)에 대한 전이확률밀도 $p^{\Delta}\left(x_{0}, x\right)$ 는, 확률과정이 $X_{0}=x_{0}$ 인 조건으로부터 $X_{\Delta}=x$ 로 변 화될 조건부 확률밀도이다. 즉,

$$
\operatorname{Pr}\left(X_{\Delta} \in d x \mid X_{0}=x_{0}\right)=p^{\Delta}\left(x_{0}, x\right) d \lambda(x)
$$

이다. 여기서 $\lambda(\cdot)$ 는 르벡척도(Lebesgue measure)이다. 확산계수가 $\sigma(x)$ 와 같이 $x$ 의 함수로 주어진 경우, $1 / \sigma(u)$ 에 대한 부정적분으로 주어지는 Lamperti 변환,

$$
h(x)=\int_{c}^{x} \frac{d u}{\sigma(u)}
$$


을 이용하여 정의되는 확산과정 $Y_{t}=h\left(X_{t}\right)$ 는 확산계수가 1 인 단위확산과정이 된다 (여기서 $c$ 는 임의 의 상수). 즉,

$$
d Y_{t}=a\left(Y_{t}\right) d t+d W_{t}
$$

이다. 여기서

$$
a(y)=a(h(x))=\frac{\mu(x)}{\sigma(x)}-\frac{1}{2} \sigma^{\prime}(x)
$$

이다.

이때 확산과정 $X_{t}$ 에 대한 전이확률밀도 $p^{\Delta}\left(x_{0}, x\right)$ 와 변환된 확산과정 $Y_{t}$ 에 대한 전이확률밀도 $q^{\Delta}\left(y_{0}\right.$, $y$ ) 사이에는 다음과 같은 관계가 성립한다.

$$
p^{\Delta}\left(x_{0}, x\right)=\frac{q^{\Delta}\left(h\left(x_{0}\right), h(x)\right)}{\sigma(x)} .
$$

이와 같이 확산과정 $X_{t}$ 에 대한 전이확률밀도는 Lamperti 변환을 통하여 얻어진 확산과정 $Y_{t}$ 에 대한 전 이확률밀도 $q^{\Delta}\left(y_{0}, y\right)$ 에 대한 식으로 표현 된다.

Shoji와 Ozaki (1998)가 제안한 NLL 추정방법은 Hurn 등 (2007)의 비교 시험 연구에서 그 단순성에 비하여 예상과 달리 좋은 성질을 보여주고 있다. NLL 추정방법은 확산모형 (3.1)에 대한 전이확률밀도 $q^{\Delta}\left(y_{0}, y\right)$ 를 구하기 위하여 확산모형의 추세계수를,

$$
a\left(Y_{t}\right) \approx a_{0}+a_{0}^{\prime}\left(Y_{t}-y_{0}\right)+\frac{1}{2} a_{0}^{\prime \prime} t
$$

라고 근사하여 그 전이확률밀도를 구한 것이다. 여기서 $a_{0}, a_{0}^{\prime}, a_{0}^{\prime \prime}$ 는 각각 $a\left(y_{0}\right), a^{\prime}\left(y_{0}\right), a^{\prime \prime}\left(y_{0}\right)$ 를 의미 한다. 이때 $a_{0}^{\prime} \neq 0$ 인 경우

$$
\begin{aligned}
& \Delta_{1}\left(a_{0}^{\prime}\right)=\frac{\left(e^{a_{0}^{\prime} \Delta}-1\right)}{a_{0}^{\prime}}, \\
& \Delta_{2}^{2}\left(a_{0}^{\prime}\right)=\frac{2\left(e^{a_{0}^{\prime} \Delta}-1-a_{0}^{\prime} \Delta\right)}{\left(a_{0}^{\prime}\right)^{2}}
\end{aligned}
$$

라 하고, $a_{0}^{\prime}=0$ 인 경우 $\Delta_{1}\left(a_{0}^{\prime}\right)=\Delta$ 이고 $\Delta_{2}^{2}\left(a_{0}^{\prime}\right)=\Delta^{2}$ 이라 하면, $\mathrm{OU}$ 모형 $d Y_{t}=\alpha\left(\beta-Y_{t}\right) d t+d W_{t}$ 에 대하여 $Y_{\Delta}$ 의 분포는 평균이 $y_{0}+a_{0} \Delta_{1}\left(a_{0}^{\prime}\right)$ 이고 분산이 $\Delta_{1}\left(2 a_{0}^{\prime}\right)$ 인 정규분포가 된다. 이를 이용하여 $\mathrm{NLL}$ 방법에서는 일반적인 확산과정 $Y_{t}$ 에 대한 전이확률밀도 $q^{\Delta}\left(y_{0}, y\right)$ 를 평균 $m(\Delta)$ 과 분산 $s^{2}(\Delta)$ 가 각각

$$
m(\Delta)=y_{0}+a_{0} \Delta_{1}\left(a_{0}^{\prime}\right)+\frac{1}{4} a_{0}^{\prime \prime} \Delta_{2}^{2}\left(a_{0}^{\prime}\right), \quad s^{2}(\Delta)=\Delta_{1}\left(2 a_{0}^{\prime}\right)
$$

와 같이 정의되는 정규분포로 근사한다. 즉,

$$
Y_{\Delta} \sim \mathcal{N}\left(m(\Delta), s^{2}(\Delta)\right)
$$

이다.

확산과정 $X_{t}$ 를 $\Delta$ 시간간격으로 관측한 값들을 $\mathbf{x}=\left\{\mathrm{x}_{0}, \mathrm{x}_{1}, \ldots, \mathrm{x}_{n}\right\}$ 라고 나타낸 것과 마찬가지로 함수 $h(\cdot)$ 로 $\mathrm{x}_{i}, i=0,1, \ldots$ 를 변환한 값들을 $\mathrm{y}_{i}$ 라 하고 $\mathbf{y}=\left\{\mathrm{y}_{0}, \mathrm{y}_{1}, \ldots, \mathrm{y}_{n}\right\}$ 이라 하자. Lamperti 변환 $h(\cdot)$ 
는 확산계수 $\sigma(\theta, \cdot)$ 의 부정적분형태로 주어지는 함수이므로, $\mathrm{y}_{i}$ 또한 추정할 모수 $\theta$ 의 함수이다. 즉, $\mathrm{y}_{i}=\mathrm{y}_{i}(\theta)$ 이다. 이후에서 표현의 간략화를 위해서 $a\left(\mathrm{y}_{i}\right), a^{\prime}\left(\mathrm{y}_{i}\right), a^{\prime \prime}\left(\mathrm{y}_{i}\right)$ 를 각각 $a_{i}, a_{i}^{\prime}, a_{i}^{\prime \prime}$ 라고 나타내기 로 하고, 또한 $\Delta_{1}\left(2 a_{i}^{\prime}\right), \Delta_{1}\left(a_{i}^{\prime}\right), \Delta_{2}^{2}\left(a_{i}^{\prime}\right)$ 를 각각 $\Delta_{0, i}, \Delta_{1, i}, \Delta_{2, i}^{2}$ 로 나타내기로 한다. 또 $\sigma\left(\theta, x_{i}\right)$ 는 $\sigma_{i}$ 로 나타내기로 하자.

위의 식 (3.5)로부터 $\mathrm{y}_{i-1}$ 가 주어진 경우 $\mathrm{Y}_{i}$ 의 분포로 다음과 같은 근사식을 고려하자.

$$
\mathrm{Y}_{i}-\mathrm{y}_{i-1} \sim \mathcal{N}\left(a_{i-1} \Delta_{1, i-1}+\frac{1}{4} a_{i-1}^{\prime \prime} \Delta_{2, i-1}^{2}, \Delta_{0, i-1}\right)
$$

이다. Lee 등 (2012)와 Lee과 Lee (2013)은 위의 근사식 (3.6)에서 분산항은

$$
\operatorname{Var}\left(\mathrm{Y}_{i}-\mathrm{y}_{i-1}\right) \simeq \Delta_{0, i-1}+\frac{2}{3}\left(a_{i-1} a_{i-1}^{\prime \prime}+\frac{1}{2} a_{i-1}^{\prime \prime \prime}\right) \Delta_{0, i-1}^{3}
$$

와 같이 확장될 수 있음을 보이고 있다. 여기에서 Lamperti 변환에 따라 단위확산과정을 얻은 이후에 오일러근사법을 적용하는 경우와의 비교를 위하여

$$
\mathrm{Y}_{i}-\mathrm{y}_{i-1} \sim \mathcal{N}\left(a_{i-1} \Delta, \Delta\right)
$$

인 경우를 함께 고려하기로 하자.

위의 근사식 (3.6)을 고려하여 $\mathrm{GMM}$ 을 적용하기 위한 대안으로 다음과 같은 방법들이 고려된다. $\mathrm{GMM}$ 을 적용하는 방법으로 관측값 $\mathrm{x}$ 를 직접 이용하여 $\mathrm{GMM}$ 을 적용하는 방법이 있고 (이하에서 NGMM-X이라 함), $\mathbf{x}$ 대신에 변환된 값 $\mathbf{y}$ 을 이용하여 GMM을 적용하는 방법을 고려할 수 있다 (이 하에서 NGMM-Y이라 함). NGMM-X의 경우에는 $\left(\mathrm{Y}_{i}-\mathrm{y}_{i-1}\right)$ 의 평균 혹은 분산이나 고차적률들에 대한 식들을 $\left(\mathrm{X}_{i}-\mathrm{x}_{i-1}\right)$ 에 대한 평균이나 적률로 변환하여야 하는 문제가 발생하고, NGMM-Y의 경 우에는 $\mathrm{y}$ 가 추정할 미지인 모수의 함수라는 점이 있어서 표본적률의 계산에 모수가 개입되게 된다. NGMM-Y와의 비교를 위하여 위의 근사식 (3.7)에 근거하여 GMM을 적용하는 방법을 NGMM-E라고 나타내기로 하자.

먼저 NGMM-Y의 경우를 살펴보자. NGMM-Y는 $\varepsilon_{i}$ 과 $v_{i}^{2}$ 을

$$
\begin{aligned}
\varepsilon_{i} & =\left(\mathrm{y}_{i}-\mathrm{y}_{i-1}\right)-a_{i-1} \Delta_{1, i-1}-\frac{1}{4} a_{i-1}^{\prime \prime} \Delta_{2, i-1}^{2}, \\
v_{i}^{2} & =\Delta_{0, i-1}
\end{aligned}
$$

와 같이 설정하고, 이를 식 (2.2)와 (2.3)에 대입하여 $Q_{n}(\theta)$ 를 정의하고 이를 최소화 하는 추정량 $\hat{\theta}$ 를 찾 는 방법이다. 위의 근사식 (3.6)이 정확한 것으로 가정하면 $E\left[\sqrt{n} \psi_{j}\right]=0, j=1,2, \ldots, J$ 이다. 이 때 분산공분산행렬 $V$ 는 식 (2.5)과 (2.6)으로부터 얻어진다.

근사식 (3.7)에 근거하여 GMM을 적용하는 방법인 NGMM-E는 다음과 같이 $\varepsilon_{i}$ 과 $v_{i}^{2}$ 을 설정하여 얻어 진다.

$$
\begin{aligned}
\varepsilon_{i} & =\left(\mathrm{y}_{i}-\mathrm{y}_{i-1}\right)-a_{i-1} \Delta, \\
v_{i}^{2} & =\Delta .
\end{aligned}
$$

이때 $\psi_{j}$ 는 $\varepsilon_{i}$ 과 $v_{i}^{2}$ 을 식 (2.2)와 (2.3)에 대입하여 얻어지고, 분산공분산행렬 $V$ 는 식 (2.5)와 (2.6)에 대입하여 얻어진다. NGMM-E는 근사식 (3.7)에 근거하여 얻어지는 것으로 NLL과는 무관하고 Lamperti 변환만을 고려한 경우이므로 NGMM이라 부르기 어려운 점이 있지만, 명칭의 통일성을 위해서 본 논문에서는 이와 같이 부르기로 한다. 
다음으로 관측값 $\mathbf{x}$ 를 직접 이용하는 NGMM-X를 살펴보자. Lamperti 변환에서의 적분항을 근사하여 다음과 같은 관계를 고려할 수 있다.

$$
\mathrm{y}_{i}-\mathrm{y}_{i-1}=\int_{\mathrm{x}_{i-1}}^{\mathrm{x}_{i}} \frac{1}{\sigma(\theta, x)} d x \simeq\left(\mathrm{x}_{i}-\mathrm{x}_{i-1}\right) \cdot\left(\frac{1}{\sigma_{i-1}}\right) .
$$

이로부터, $i=1,2, \ldots$ 에 대하여

$$
\mathrm{X}_{i}-\mathrm{x}_{i-1} \sim \mathcal{N}\left(\sigma_{i-1} a_{i-1} \Delta_{1, i-1}+\frac{1}{4} \sigma_{i-1} a_{i-1}^{\prime \prime} \Delta_{2, i-1}^{2}, \sigma_{i-1}^{2} \Delta_{0, i-1}\right)
$$

라고 근사할 수 있고, 식 (3.2)로부터, $i=0,1, \ldots$ 에 대하여

$$
\begin{aligned}
& a_{i}=\left(\frac{\mu_{i}}{\sigma_{i}}\right)-\frac{1}{2} \sigma_{i}^{\prime}, \\
& a_{i}^{\prime}=\mu_{i}^{\prime}-\sigma_{i}^{\prime}\left(\frac{\mu_{i}}{\sigma_{i}}\right)-\frac{1}{2} \sigma_{i} \sigma_{i}^{\prime \prime}, \\
& a_{i}^{\prime \prime}=\sigma_{i} \mu_{i}^{\prime \prime}-\mu_{i}^{\prime} \sigma_{i}^{\prime}+\left\{\left(\sigma_{i}^{\prime}\right)^{2}-\sigma_{i} \sigma_{i}^{\prime \prime}\right\}\left(\frac{\mu_{i}}{\sigma_{i}}\right)-\frac{1}{2} \sigma_{i} \sigma_{i}^{\prime} \sigma_{i}^{\prime \prime}-\frac{1}{2} \sigma_{i}^{2} \sigma_{i}^{(3)}
\end{aligned}
$$

임을 얻는다. 여기서 $\mu^{\prime}, \mu^{\prime \prime}, \sigma^{\prime}, \sigma^{\prime \prime}, \sigma^{(3)}$ 은 각기 추세계수 $\mu(x)$ 와 확산계수 $\sigma(x)$ 를 $x$ 에 대하여, 1 차, 2 차, 3 차 미분한 함수를 의미한다. 위에서 $a$ 는 $y$ 의 함수, 즉 $a(y)$ 이고, $\mu$ 와 $\sigma$ 는 $x$ 의 함수 즉 $\mu(x)$, $\sigma(x)$ 이므로, $a$ 에 적용된 첨자 $i$ 는 $y$ 값으로 $\mathrm{y}_{i}$ 가 대입되었음을 의미하고, $\mu$ 와 $\sigma$ 에 적용된 첨자 $i$ 는 $x$ 값 에 $\mathrm{x}_{i}$ 가 대입되었음을 의미한다. 즉, $a_{i}^{\prime}=a^{\prime}\left(\mathrm{y}_{i}\right)$ 이고 $\mu_{i}^{\prime}=\mu^{\prime}\left(\mathrm{x}_{i}\right)$ 인 의미이다. 이로부터 $\varepsilon_{i}$ 와 $v_{i}^{2}$ 을 다음 과 같이 설정한다.

$$
\begin{aligned}
\varepsilon_{i} & =\left(\mathrm{x}_{i}-\mathrm{x}_{i-1}\right)-\sigma_{i-1} a_{i-1} \Delta_{1, i-1}-\frac{1}{4} \sigma_{i-1} a_{i-1}^{\prime \prime} \Delta_{2, i-1}^{2}, \\
v_{i}^{2} & =\sigma_{i-1}^{2} \Delta_{0, i-1} .
\end{aligned}
$$

또 $\psi_{j}, j=1,2, \ldots, J$ 는 이를 식 (2.2)와 (2.3)에 대입하여 얻고, 이 때 분산공분산행렬 $V$ 는 식 (2.5)와 (2.6)으로 부터 얻는다.

\section{4. 도구변수의 선택, 고차적률, 비일치성, CKLS 모형에 대한 고려}

$\mathrm{GMM}$ 은 기본적으로 확률적변량 $\varepsilon_{i}$ 와 다른 도구변수 $z_{i, j}$ 사이의 독립성을 이용한 추정방법이다. 변수 들 사이의 독립성을 이용하여 가설검정을 하는 독립성검정법과 마찬가지로 GMM은 변수들 사이의 독 립성을 이용하여 통계적 모형의 모수를 추정하는 것이다. 독립성검정 방법이 동일성검정이나 적합성결 여검정 등 다양한 형태로 이용되는 것과 마찬가지로 GMM 또한 다양하고 유연성 있는 형태로 변형 적 용이 가능하다. 그 한 예가 공간통계학 분야에서 고려되는 베리오그램 추정법이다. 공간적으로 관측되 는 변량들 사이의 자기상관성을 표현하는 베리오그램을 추정하기 위한 방법으로 이용되는 최소자승법은 정확하게 $\mathrm{GMM}$ 의 한 형태이다.

이런 이유로 도구변수는 $\varepsilon_{i}$ 와 독립성을 갖는 임의의 변량이면 적용이 가능하다. 그러므로 비확률적변 량의 경우는 어떤 값이든 상관없이 도구변수로 사용될 수 있다. 그러나 여러 가지 다양한 형태의 도구 변수들 중 $\mathrm{GMM}$ 의 장점이 돋보이게 하기 위해서는 그 도구변수의 선택과 사용이 용이해야 하며, 통계 적 효율성 또한 높아야 하고 이에 더하여 수치적 계산 또한 용이해야 한다. 일반적인 통계적 추정이론에 
서 추정량의 통계적 효율성을 높이기 위해서는 도구변수에 해당하는 변수들에 대하여 $z_{i, j}=\left(\partial / \partial \theta_{j}\right) \varepsilon_{i}$, $j=1,2, \ldots, J_{1}$ 이고 $z_{i, J_{1}+j}=\left(\partial / \partial \theta_{j}\right)\left(\varepsilon_{i}^{2}-v_{i}^{2}\right), j=1,2, \ldots, J_{2}$ 임이 알려져 있다. 여기서 $J_{1}$ 은 $\varepsilon_{i}$ 에 개 입되어 있는 모수의 개수이고, $J_{2}$ 은 $\left(\varepsilon_{i}^{2}-v_{i}^{2}\right)$ 에 개입되어 있는 모수의 개수이다 (Heyde, 1997; Lahiri 등, 2002; Lee와 Lahiri, 2002). 최대우도추정법에 따르는 정규방정식(normal equation)을 살펴보면 최 적의 도구변수를 쉽게 찾을 수 있다. CKLS 모형에 대하여 오일러근사법에 근거하여 최대우도추정량을 구하는 경우를 고려하여 도구변수의 대안을 살펴보면 $1, x_{i}, \log \left(x_{i}\right)$ 이 고려될 수 있다. 여기서 $\log \left(x_{i}\right)$ 의 경우 $x_{i}$ 가 0 혹은 음의 값을 갖는 경우 계산에 문제가 발생한다. 또한 GMM의 경우에 있어서 도구변수 의 선택은 사용의 용이성이 중요한 역할을 하는 점이 있으므로 본 연구에서는 이런 점을 고려하여, 계산 의 용이성과 통계적 효율성을 함께 고려하여 도구변수로, 모든 원소가 1 인 벡터 1 과 함께 다음과 같이 정의하여 서로 직교가 되는 두 벡터 $\tilde{\mathbf{x}}^{(1)}=\left(\tilde{\mathrm{x}}_{0}^{(1)}, \ldots, \tilde{\mathrm{x}}_{n-1}^{(1)}\right)^{t}$ 와 $\tilde{\mathbf{x}}^{(2)}=\left(\tilde{\mathrm{x}}_{0}^{(2)}, \ldots, \tilde{\mathrm{x}}_{n-1}^{(2)}\right)^{t}$ 를 이용한다.

$$
\begin{aligned}
& \tilde{\mathrm{x}}_{i}^{(1)}=\mathrm{x}_{i}-u, \\
& \tilde{\mathrm{x}}_{i}^{(2)}=\left(\mathrm{x}_{i}-u\right)^{2}-d_{2}-\left(\frac{d_{3}}{d_{2}}\right)\left(\mathrm{x}_{i}-u\right),
\end{aligned}
$$

여기서 $i=0,1, \ldots,(n-1)$ 이고,

$$
\begin{aligned}
u & =\frac{1}{n} \sum_{i=0}^{n-1} \mathrm{x}_{i}, \\
d_{2} & =\frac{1}{n} \sum_{i=0}^{n-1}\left(\mathrm{x}_{i}-u\right)^{2}, \\
d_{3} & =\frac{1}{n} \sum_{i=0}^{n-1}\left(\mathrm{x}_{i}-u\right)^{3}
\end{aligned}
$$

이다. 이와 같이 도구변수가 직교가 되도록 잡는 이유는 분산공분산행렬 $V$ 가 대각행렬이 되고 그 역행 렬로 주어지는 가중행렬 $W$ 의 계산이 용이하도록 하기 위해서이다 (Imbens 등, 1998). NGMM의 경우 에 있어서는 행렬 $V$ 의 비대각원소가 정확하게 0 이 되지는 않으나, 전체적으로 그 영향을 무시할 수 있 다고 보아 마찬가지로 대각원소들만을 고려하기로 한다.

$\mathrm{GMM}$ 추정량이 일치성을 갖기위해서는 $E\left[\psi_{j}\right]=0, j=1,2, \ldots, J$ 를 만족해야 한다. 만약 이 조건 이 만족되지 않는 경우 추정량은 일치성을 갖지 않는다. 만약 $E\left[\psi_{j}\right]=\delta \neq 0$ 인 경우 식 (2.4)에서 $\mathbf{m}$ 은 $O(\sqrt{n} \delta)$ 이 되므로, 표본의 크기 $n$ 이 커지더라도 MSE는 0 으로 수렴하지 않는다. 만약 근사적 모 형 (3.6)이 사실임에도 불구하고 오일러근사법에 기반한 EGMM을 적용하는 경우를 고려해 보자. 이런 경우에는 전이확률분포에서 평균의 불일치 문제가 발생하므로 표본의 크기 $n$ 만을 크게 잡는 방법으로는 일치성을 갖는 추정량을 얻을 수 없다. 실제로는 NLL에 근거하여 전이확률분포를 근사한 식 (3.6) 마 저도 정확한 전이확률밀도와 일치하는 것이 아니므로 오일러근사법을 적용하는 EGMM 방법뿐만 아니 라 NGMM-Y 그리고 NGMM-X 또한 일치성을 주는 추정량을 주지 못한다. 이런 문제를 해결하기 위 해서는 표본의 크기 $n$ 과 함께 관측시간간격 $\Delta$ 또한 0 으로 수렴하는 상황에서의 점근성을 고려해야 한 다. $\Delta$ 가 0 으로 수렴하는 것을 가정하는 경우 NLL에 기반한 근사식 (3.6)이나 오일러근사법에서 얻어 지는 식 (2.1)이 정확한 전이확률밀도와 같아지게 되므로 추정량의 비일치성문제가 해결된다. 도구변수 의 설정과 $\mathrm{GMM}$ 의 점근성 문제에 대하여는 저자들이 별도의 연구 논문을 작성 중이기도 하다.

앞서 정의된 $\psi_{j}$ 들은 $\varepsilon_{i}$ 혹은 $\varepsilon_{i}^{2}$ 의 함수로서만 정의되었다. $\mathrm{GMM}$ 적용방법의 유연성에 기초하여 $\psi_{j}$ 를 $\varepsilon_{i}^{3}$ 혹은 $\varepsilon_{i}^{4}$ 의 함수로 정의하여 전이확률밀도의 고차적률에 관한 식을 포함시킬 수도 있다. Lee과 Lee 
(2013)은

$$
\begin{aligned}
& E\left[\varepsilon_{i}^{3}\right] \simeq a_{i-1}^{\prime \prime} \Delta^{3}, \\
& E\left[\varepsilon_{i}^{4}\right] \simeq a_{i-1}^{(3)} \Delta^{4}
\end{aligned}
$$

임을 보이고 있다. 이 관계를 이용하여 NGMM-Y의 경우

$$
\psi_{j}(Z ; \theta)=\frac{1}{n} \sum_{i=1}^{n} z_{i, j}\left(\varepsilon_{i}^{3}-a_{i-1}^{\prime \prime} \Delta^{3}\right)
$$

혹은

$$
\psi_{j}(Z ; \theta)=\frac{1}{n} \sum_{i=1}^{n} z_{i, j}\left(\varepsilon_{i}^{4}-a_{i-1}^{(3)} \Delta^{4}\right)
$$

을 포함하도록 하는 방법이 고려될 수 있다.

CKLS 모형은 $\mu(\theta, x)=\alpha(\beta-x)$ 이고 $\sigma(\theta, x)=\sigma x^{\gamma}$ 이므로, 이에 대한 Lamperti 변환은

$$
y=h(x)=\frac{1}{\sigma} \int_{c}^{x} x^{-\gamma} d x
$$

이고, 정적분 구간의 하한 $c$ 의 선택은 단위확산 $Y_{t}$ 의 확률적성질에 어떤 영향도 미치지 않는다. 왜냐하 면, 실제 전이확률밀도함수에 영향을 미치는 값은 개별적인 $\mathrm{y}_{i}$ 값이 아니고 그 차분값들인 $\left(\mathrm{y}_{i}-\mathrm{y}_{i-1}\right)$ 이 므로 적분의 하한은 이에 영향을 미치지 못하고, $y$ 값이 전이확률밀도에 영향을 미치는 또 다른 통로는 오직 함수 $a(\cdot)$ 와 그 미분함수들을 통해서만이다. 그런데, 식 (3.2)인 관계에 의하여, $a(y)$ 와 이의 (고 차)미분함수들은, 모두 Lamperti 변환의 영향을 받지 않는 $x$ 의 식으로 표현되므로 결국 $c$ 는 아무런 영 향을 미치지 못한다.

보통 Lamperti 변환에서 $c=0$ 인 경우가 자주 고려되고 이에 대응하는 $h(x)=x^{1-\gamma} /(\sigma(1-\gamma))$ 인 멱변 환함수가 자주 고려되나 이때 $\gamma=1$ 인 경우에 $h(x)$ 가 정의되지 않는 문제가 발생하여 개별적인 변환값 $y$ 를 잡을 수 없는 문제가 발생한다. 이런 문제점을 피하기 위하여 $c=1$ 인 경우를 고려하면 Lamperti 변환은 다음과 같이 Box-Cox 변환의 형태로 표현된다.

$$
h(x)= \begin{cases}\frac{1}{\sigma} \frac{\left(x^{1-\gamma}-1\right)}{(1-\gamma)}, & \gamma \neq 1 \text { 일 때, } \\ \frac{1}{\sigma} \log (x), & \gamma=1 \text { 일 때 }\end{cases}
$$

이다. 이때 $y=h(x)$ 에 의하여 변환된 $y$ 값은 $\gamma$ 값의 범위에 관계없이 $x$ 값이 증가하면 따라서 증가하는 성질을 갖는다. 그러나 $\gamma$ 가 1 보다 큰 값을 갖게 되는 경우에서 $x^{1-\gamma}$ 항의 계수는 음수의 형태로 나타난 다. 즉, $\gamma=1.5$ 인 경우 $y=2(1-1 / \sqrt{x})$ 가 된다. CKLS 모형에서 변환된 단위확산과정 $Y_{t}$ 의 추세계 수와 그 미분함수들은 다음과 같이 $x$ 의 함수로 표현된다.

$$
\begin{aligned}
a(y) & =\left(\frac{\alpha}{\sigma}\right)(\beta-x) x^{-\gamma}-\frac{1}{2} \sigma \gamma x^{\gamma-1}, \\
a^{\prime}(y) & =\alpha(\gamma-1)-\alpha \beta \gamma x^{-1}-\frac{1}{2} \sigma^{2} \gamma(\gamma-1) x^{2 \gamma-2}, \\
a^{\prime \prime}(y) & =\sigma \alpha \beta \gamma x^{\gamma-2}-\sigma^{3} \gamma(\gamma-1)^{2} x^{3 \gamma-3}, \\
a^{\prime \prime \prime}(y) & =\sigma^{2} \alpha \beta \gamma(\gamma-2) x^{2 \gamma-3}-3 \sigma^{4} \gamma(\gamma-1)^{3} x^{4 \gamma-4} .
\end{aligned}
$$




\section{5. 모의실험에 의한 비교}

앞에서 제시된 네 개의 GMM 추정량 EGMM, NGMM-E, NGMM-Y, NGMM-X와 오일러근사법에 의 한 추정량 Euler 그리고 NLL 추정법들의 성질을 비교하기 위하여, CKLS 모형을 중심으로 모의실험 연구를 수행한다. OU 모형 CIR 모형은 CKLS 포함되는 측면이 있고, 특히 OU 모형의 경우는 비교대 상이 되는 추정방법들의 성능에 큰 차이가 없으므로 본 논문에서는 비교기준이 되는 모형에 포함하지 않 고 CKLS 모형을 중심으로 모의실험을 수행한다.

전이확률밀도함수가 잘 알려져 있어서 최대우도추정법의 적용이 가능한 $\mathrm{CIR}$ 모형의 경우와 달리 CKLS 모형의 경우에 대하여는 최대우도 추정법을 적용하는데 어려움이 따른다. Aït-Sahalia (1999), Aït-Sahalia (2002), Lee 등 (2012)는 확산모형의 우도함수를 구하는 방법에 관하여 연구하고 있고, 이 를 이용하면 CKLS 모형 등에 대한 최대우도추정법의 적용이 가능하다.그러나 상대적으로 복잡한 계산 절차가 필요하다. CKLS 모형에서 모수 $\gamma$ 의 값이 0.5 인 경우, 즉 CKLS 모형이 CIR 모형과 같아지는 경우에는 정확한 우도함수 계산을 위한 복잡한 계산을 거치지 않고서도, 모의실험을 하는 경우에, 미리 $\gamma$ 의 실제값이 0.5 인 것을 안다고 가정하고 나머지 모수에 대하여 CIR 모형에서의 최대우도추정법을 적 용하는 방법을 생각해 볼 수 있다. 이는 정확한 최대우도추정법과는 의미가 다르나, 어느 정도 최대우도 추정법과 유사성을 가질 것이므로 이를 다른 추정법들과 비교해보는 것은 나름대로 의미있는 비교가 될 것이다. 이와 같이 $\gamma$ 의 실제값이 0.5 인 것을 안다고 가정하고 수행한 최대우도추정법을 가정된 최대우 도추정법(Assumed Maximum Likelihood Estimator; AMLE)라고 부르기로 하고 다른 추정방법들과 함께 비교하기로 한다.

모의실험에서 사용된 모수 $\theta=(\alpha, \beta, \sigma, \gamma)$ 값은 $(0.15,0.04,0.05,0.5)$ 인 경우를 시험하였다. 이는 보통 이자율 모형화 등에서 고려되는 경우들에서 가정되는 모수값과 비슷한 경우이다. 표본 생성을 위해서는 각 관측치들 사이의 시간 간격 혹은 $\Delta$ 를 $1 / 4$ 인 경우와 $1 / 12$ 인 경우를 상정하였고, 연속시간 확률과정 을 모사하여 생성하기 위하여 $\Delta$ 시간간격을 Hurn 등 (2007)에서와 마찬가지 방법으로 더욱 작은 시간 단위로 분할하여 오일러 방법에 따라 확산과정을 생성하고 그 중에서 일정간격으로 표본의 관측값을 취 하는 방법을 사용하였다. 본 연구의 모의실험에서는 시간단위를 100 개의 작은 시간단위로 분할하여 관 측값을 생성하였다. 또한 표본의 크기는 100 인 경우와 400 인 경우를 시험하였다.

Table 5.1은 각각 CKLS 모형에 대한 1000 회의 시뮬레이션 결과를 요약한 표이다. 표에서 제시된 값은 $\theta=(\alpha, \beta, \sigma, \gamma)$ 에 대한 추정량 $\hat{\theta}$ 의 시뮬레이션 평균값, 또 오차제곱평균의 제곱근(Root Mean Square Error; RMSE), 즉 $(\hat{\theta}-\theta)^{2}$ 에 대한 시뮬레이션 평균의 제곱근이다. AMLE는 모수 $\gamma$ 에 대한 참값이 0.5 임을 알고 추정을 하는 것이므로 그에 대한 RMSE는 항상 0 으로 나타난다. Table 5.1에 정리된 시 뮬레이션 결과를 살펴보면 전반적으로 Euler 추정법이나 NLL 추정법은 RMSE가 크지 않고 안정적인 특성을 보이는데 반하여, EGMM과 NGMM-X는 매우 큰 RMSE 값을 보이고 있다. 특히 확산계수와 관계된 모수 $\sigma$ 와 $\gamma$ 의 추정에서는 추정량으로서의 성질이 매우 떨어짐을 볼 수 있다. GMM 중에서는 NGMM-Y와 NGMM-E가 안정적인 특성을 갖고 있음을 보이고 있다. 표본의 크기가 작은 경우 $\alpha$ 값의 추정에 대하여는 NGMM-E가 NGMM-Y에 비하여 장점을 가지고 있고, 표본의 크기가 커지는 경우나 $\sigma$ 와 $\gamma$ 의 추정에 대하여는 NGMM-Y가 더 좋은 성질을 가지고 있음을 볼 수 있다. 이때 주목할 만한 사 항은 표본의 크기가 커지거나 관측시간 간격 $\Delta$ 가 늘어나면 추정량의 편기(bias)와 RMSE가 줄어든다 는 점이다. 이는 Kim과 Lee (2011)에서도 관찰되는 사항으로 특히 점근성의 해석 문제에서 주목하여야 할 부분이다. 관측시간 간격 $\Delta$ 가 줄어들면 $\mathrm{RMSE}$ 가 줄어들 것으로 예상이 됨에도 불구하고, 얼핏보아 이와 반대의 현상이 나타나는 것은, 관측시간 간격과 표본의 개수가 서로 연동되어 있기 때문이다. 전체 관측기간이 정해진 경우에, 관측시간 간격이 줄어든다는 것은 표본의 크기가 늘어난다는 의미이다. 그 
Table 5.1. Means and RMSEs of estimator of the CKLS model parameters (In cases of 100 or 400 for the sample size $n$ and $1 / 12$ or $1 / 4$ for the observation time interval $\Delta$ are tested in the simulation experiments. The true value of the parameter $(\alpha, \beta, \sigma, \gamma)$ is $(0.15,0.04,0.05,0.5)$.)

\begin{tabular}{|c|c|c|c|c|c|c|c|c|c|c|}
\hline \multirow{2}{*}{$n$} & \multirow{2}{*}{$\Delta$} & \multirow{2}{*}{$\begin{array}{c}\text { Estimation } \\
\text { Method }\end{array}$} & \multicolumn{4}{|c|}{ Mean } & \multicolumn{4}{|c|}{ RMSE } \\
\hline & & & $\alpha$ & $\beta$ & $\sigma$ & $\gamma$ & $\alpha$ & $\beta$ & $\sigma$ & $\gamma$ \\
\hline \multirow{14}{*}{100} & \multirow{7}{*}{$1 / 12$} & Euler & 0.752 & 0.053 & 0.076 & 0.483 & 0.818 & 0.157 & 0.109 & 0.315 \\
\hline & & NLL & 0.787 & 0.049 & 0.080 & 0.493 & 0.880 & 0.117 & 0.110 & 0.314 \\
\hline & & AMLE & 0.806 & 0.078 & 0.050 & 0.500 & 0.903 & 0.506 & 0.004 & 0.000 \\
\hline & & EGMM & 0.761 & 0.077 & 0.463 & 0.138 & 0.819 & 0.369 & 2.814 & 0.728 \\
\hline & & NGMM-E & 0.718 & 0.061 & 0.103 & 0.465 & 0.780 & 0.284 & 0.174 & 0.485 \\
\hline & & NGMM-Y & 0.767 & 0.055 & 0.117 & 0.456 & 0.900 & 0.133 & 0.353 & 0.483 \\
\hline & & NGMM-X & 0.701 & 0.173 & 0.401 & 0.397 & 0.779 & 0.547 & 0.921 & 0.458 \\
\hline & \multirow{7}{*}{$1 / 4$} & Euler & 0.327 & 0.042 & 0.060 & 0.489 & 0.260 & 0.040 & 0.051 & 0.229 \\
\hline & & NLL & 0.347 & 0.041 & 0.068 & 0.513 & 0.292 & 0.043 & 0.065 & 0.227 \\
\hline & & AMLE & 0.349 & 0.049 & 0.050 & 0.500 & 0.292 & 0.243 & 0.004 & 0.000 \\
\hline & & EGMM & 0.336 & 0.121 & 1.354 & 0.505 & 0.266 & 1.478 & 6.291 & 0.736 \\
\hline & & NGMM-E & 0.313 & 0.043 & 0.085 & 0.562 & 0.253 & 0.051 & 0.095 & 0.265 \\
\hline & & NGMM-Y & 0.337 & 0.042 & 0.078 & 0.535 & 0.288 & 0.074 & 0.080 & 0.246 \\
\hline & & NGMM-X & 0.385 & 0.130 & 0.272 & 0.397 & 0.355 & 0.427 & 0.696 & 0.378 \\
\hline \multirow{14}{*}{400} & \multirow{7}{*}{$1 / 12$} & Euler & 0.289 & 0.042 & 0.052 & 0.497 & 0.209 & 0.040 & 0.020 & 0.107 \\
\hline & & NLL & 0.294 & 0.042 & 0.054 & 0.504 & 0.216 & 0.028 & 0.020 & 0.105 \\
\hline & & AMLE & 0.295 & 0.042 & 0.050 & 0.500 & 0.216 & 0.033 & 0.002 & 0.000 \\
\hline & & EGMM & 0.300 & 0.060 & 1.495 & 0.648 & 0.222 & 1.749 & 4.260 & 0.808 \\
\hline & & NGMM-E & 0.280 & 0.043 & 0.059 & 0.525 & 0.205 & 0.051 & 0.027 & 0.125 \\
\hline & & NGMM-Y & 0.286 & 0.043 & 0.058 & 0.518 & 0.212 & 0.034 & 0.025 & 0.119 \\
\hline & & NGMM-X & 0.337 & 0.102 & 0.208 & 0.372 & 0.273 & 0.343 & 0.634 & 0.345 \\
\hline & \multirow{7}{*}{$1 / 4$} & Euler & 0.185 & 0.040 & 0.049 & 0.490 & 0.073 & 0.007 & 0.014 & 0.084 \\
\hline & & NLL & 0.191 & 0.040 & 0.052 & 0.503 & 0.079 & 0.007 & 0.015 & 0.081 \\
\hline & & AMLE & 0.190 & 0.040 & 0.050 & 0.500 & 0.079 & 0.007 & 0.002 & 0.000 \\
\hline & & EGMM & 0.192 & 0.040 & 3.891 & 1.308 & 0.082 & 0.009 & 9.545 & 1.094 \\
\hline & & NGMM-E & 0.179 & 0.040 & 0.056 & 0.526 & 0.072 & 0.007 & 0.018 & 0.093 \\
\hline & & NGMM-Y & 0.186 & 0.040 & 0.054 & 0.511 & 0.078 & 0.007 & 0.017 & 0.088 \\
\hline & & NGMM-X & 0.231 & 0.059 & 0.095 & 0.311 & 0.133 & 0.126 & 0.251 & 0.322 \\
\hline
\end{tabular}

럼에도 불구하고 표본의 크기는 일정하게 둔 채 관측시간 간격을 줄인다는 것은 전체관측 기간을 줄인다 는 의미이므로 이런 이유로 당연히 RMSE가 커지게 된다. 이와 같은 현상은 공간통계학 분야에서 사용 되는 추정량들에서도 마찬가지로 발생한다 (Lahiri, 1996).

Figure 5.1, Figure 5.2, Figure 5.3, Figure 5.4는 제시된 Table 5.1에서 표본의 크기 $n$ 이 400 이고 관측시 간 간격 $\Delta$ 가 $1 / 12$ 인 경우에서 모수 $\alpha, \beta \sigma, \gamma$ 에 대한 시뮬레이션 추정값들에 대한 상자그림이다. 상자 그림을 그리는데 사용한 소프트웨어의 특성상 NGMM-Y를 NGMM.Y와 같이 '- '를 '. '으로 대체하여 나타내었다. $\sigma$ 와 $\gamma$ 의 추정에 있어서 NGMM-Y나 NGMM-E는 Euler 방법이나 NLL 방법과 매우 유사 한 특성을 보이고 있으나, EGMM과 NGMM-X는 매우 큰 RMSE를 갖는 것을 상자 그림으로도 확인할 수 있다. NGMM-X의 경우 $\beta$ 와 $\sigma$ 의 추정에 있어서 특히 그 성질이 좋지 못하였고, $\mathrm{EGMM}$ 은 $\sigma$ 와 $\gamma$ 의 추정에 있어서 그 성질이 매우 좋지 못하였다. 전통적으로 자주 사용되는 $\mathrm{GMM}$ 추정량인 $\mathrm{EGMM}$ 의 성질이 특별히 나쁜 것은 $\mathrm{EGMM}$ 이 확산계수 $\sigma(x)$ 가 상수가 아닌 경우, 즉 전이확률분포가 정규분포로 잘 근사되지 않는 경우에 그 사용에 매우 주의하여야 하는 그런 통계량이라는 점을 의미한다. 또한 $\alpha$ 의 


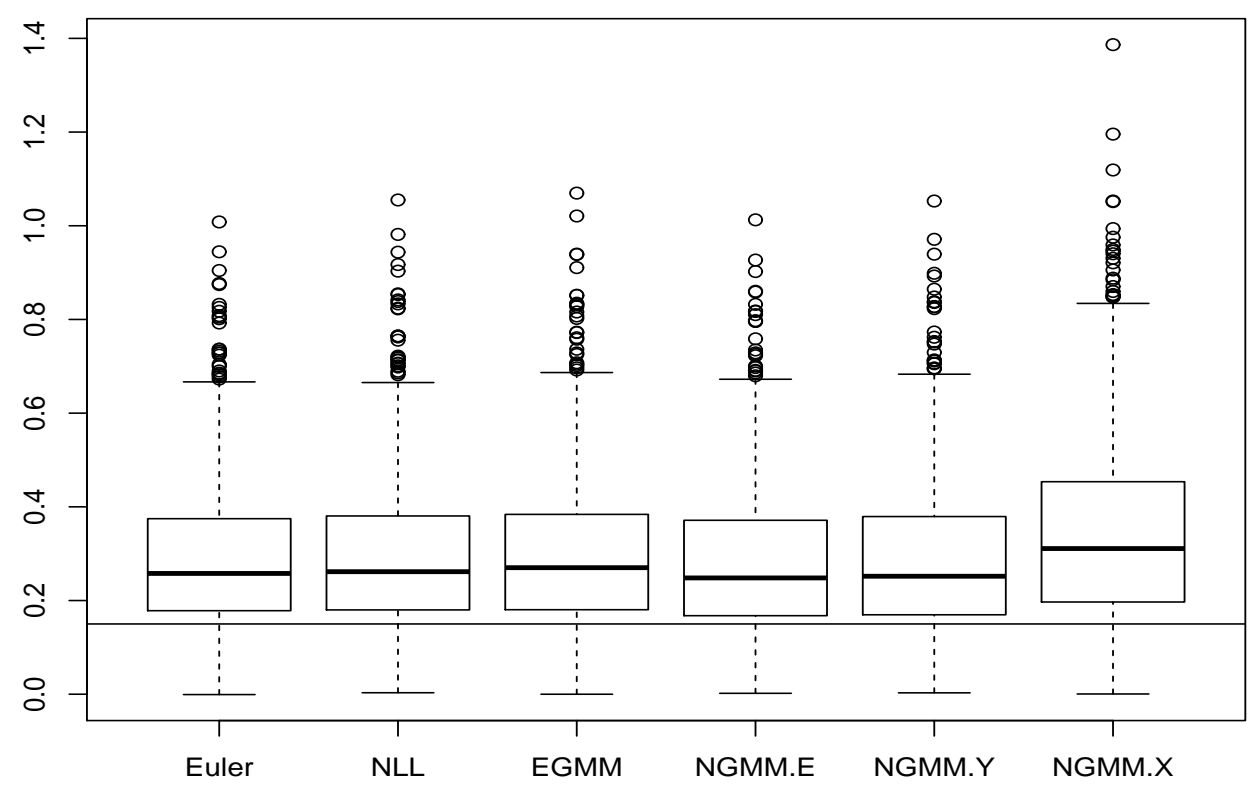

Figure 5.1. Boxplots for the estimators of the parameter $\alpha$ of the CKLS model (This boxplots show the case of 400 for the sample size $n$ and $1 / 12$ for the observation time interval $\Delta$. The solid line in the middle of the plot means the true value 0.15 of the parameter $\alpha$.)

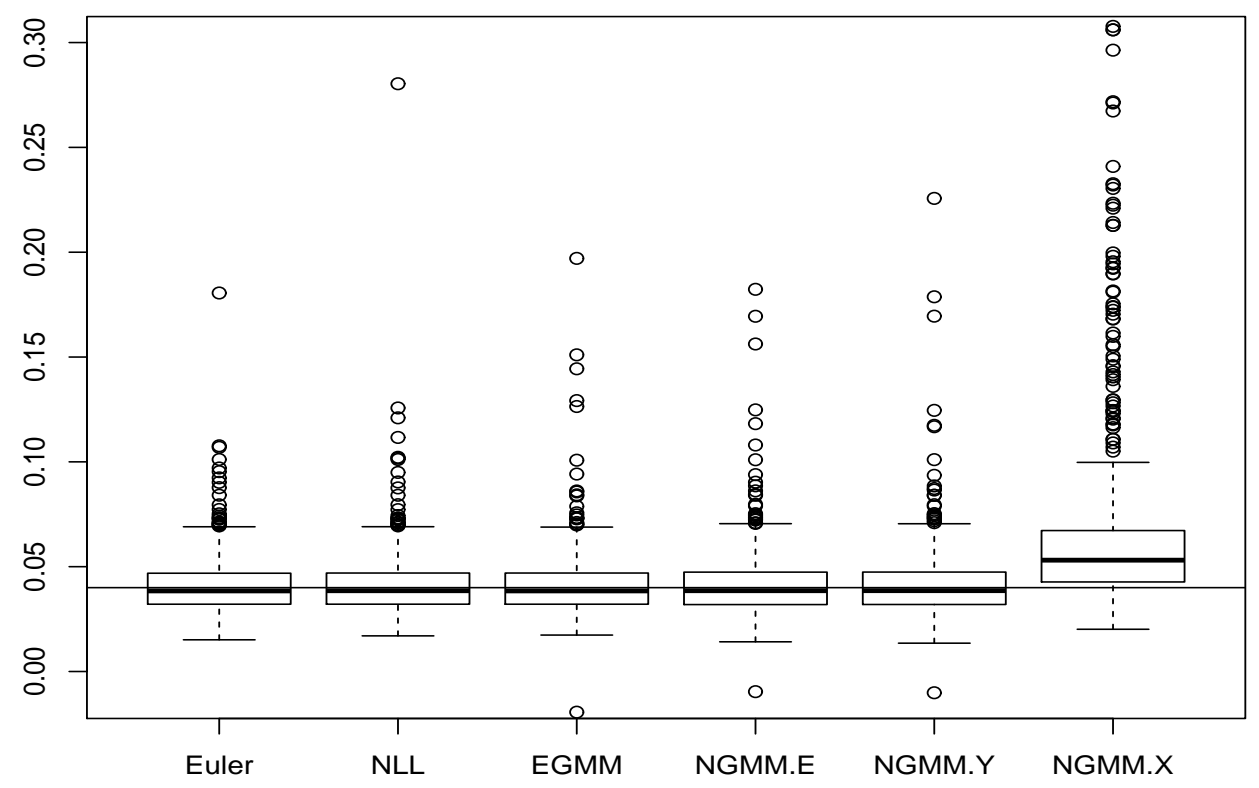

Figure 5.2. Boxplots for the estimators of the parameter $\beta$ of the CKLS model (This boxplots show the case of 400 for the sample size and $1 / 12$ for the observation time interval $\Delta$. The solid line in the middle of the plot means the true value 0.04 of the parameter $\beta$.) 


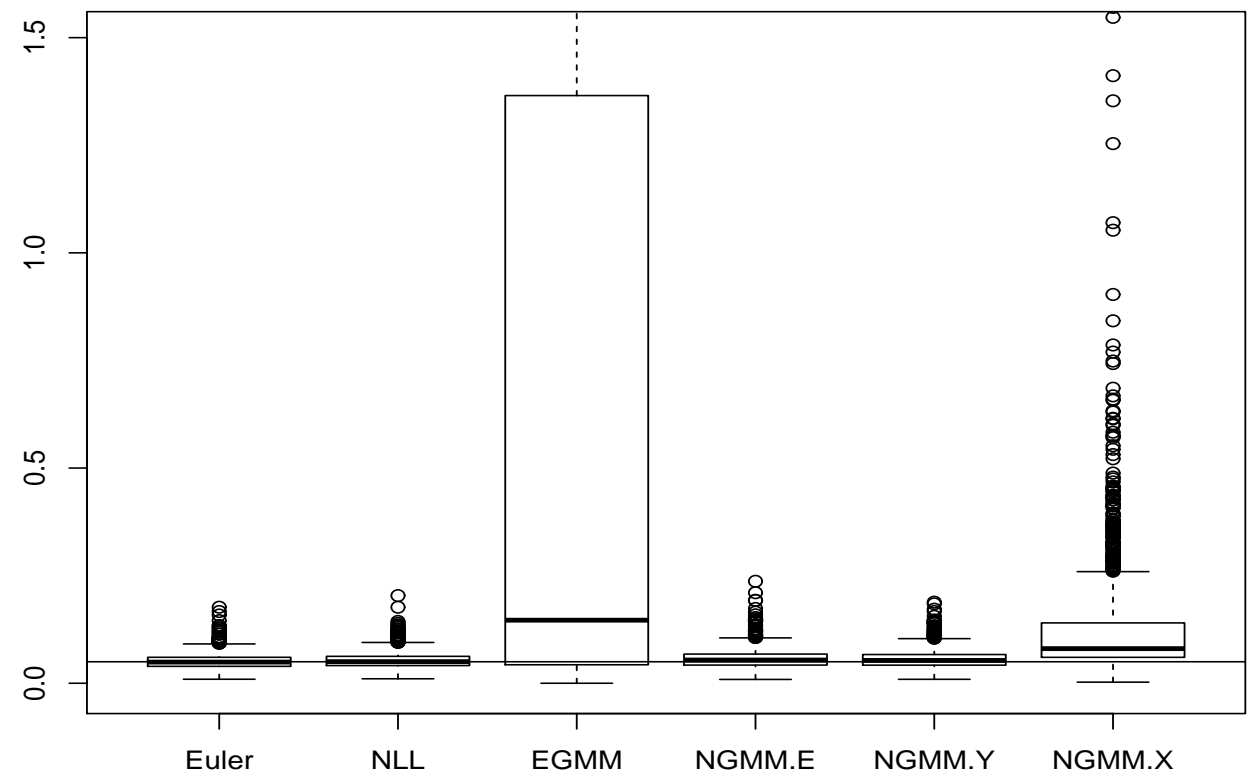

Figure 5.3. Boxplots for the estimators of the parameter $\sigma$ of the CKLS model (This boxplots show the case of 400 for the sample size $n$ and $1 / 12$ for the observation time interval $\Delta$. The solid line in the middle of the plot means the true value 0.05 of the parameter $\sigma$.)

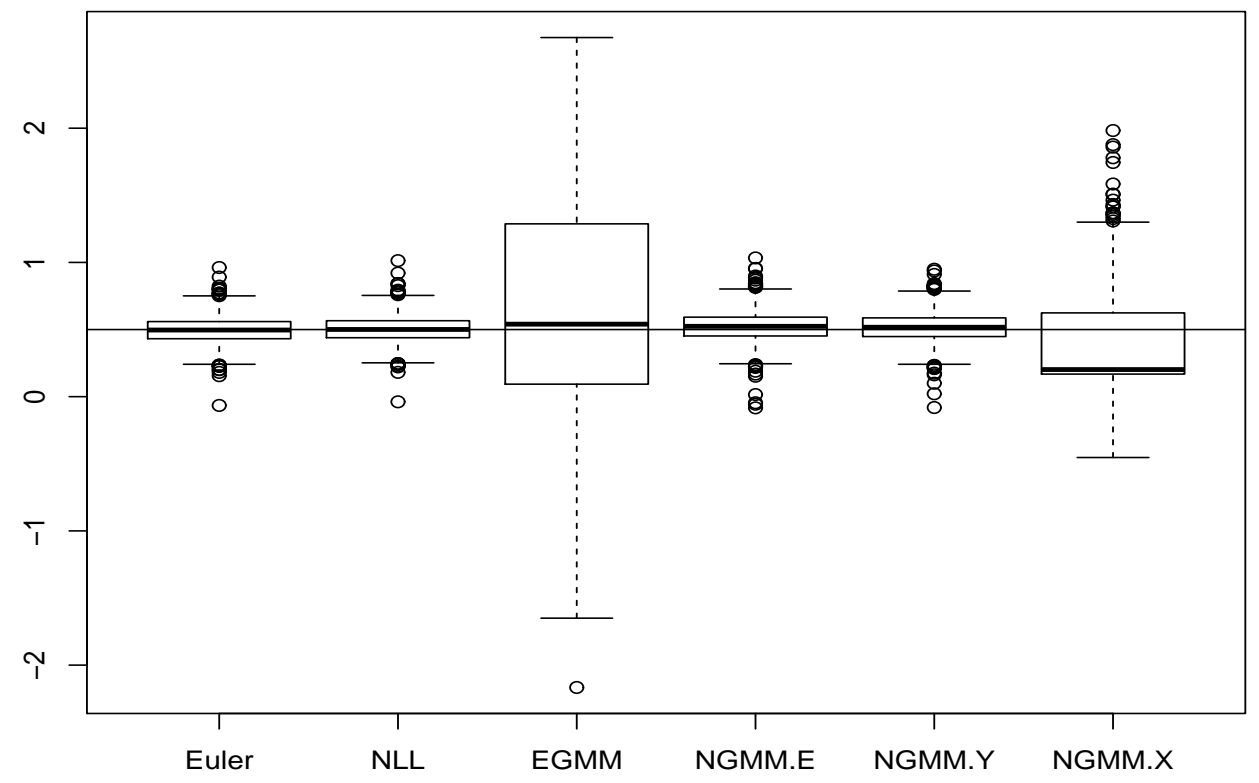

Figure 5.4. Boxplots for the estimators of the parameter $\gamma$ of the CKLS model (This boxplots show the case of 400 for the sample size $n$ and $1 / 12$ for the observation time interval $\Delta$. The solid line in the middle of the plot means the true value 0.5 of the parameter $\gamma$.) 
추정에 대하여는 시험된 모든 추정량들이 상당한 편기를 보이고 있는데 이는 특별히 $\alpha$ 추정량이 갖는 $n$ 과 $\Delta$ 의 변화에 대한 참값으로의 수렴 속도가 매우 느린 이유인 것으로 보인다. 이에 대하여는 $\alpha$ 추정 량에 대한 점근적 확장식을 깊이 있게 살펴보고 대안을 살펴보아야 할 문제인 것으로 보인다.

\section{6. 결론}

$\mathrm{GMM}$ 은 확산모형의 추정법 중에서 가장 자주 사용되는 추정법 중 하나이다. 모형의 정확한 설정없이 도 직관적으로 추정량을 구할 수 있고, 그 계산이 용이한 장점이 있다. 그러나 GMM 추정법은 CIR 모 형이나 CKLS 모형과 같이 확산계수가 상수가 아닌 확산모형의 추정에 있어서는 그 통계적 성능이 매우 좋지 못함이 잘 알려져 있다.

본 연구에서는 이와 같이 확산계수에 관여되는 모수의 추정에 있어서 $\mathrm{GMM}$ 을 적용하기 위하여 다양 한 GMM 개선 대안을 찾아보았다. 살펴본 대안들 중에서 기존에 사용되어온 EGMM과 개선 대안 중 하나인 NGMM-X는 그 통계적 성능이 다른 추정방법들에 비하여 매우 좋지 못함을 확인할 수 있었고, Lamperti 변환을 적용한 이후에 GMM을 적용한 NGMM-E와 여기에 NLL 근사법의 아이디어를 함께 적용한 NGMM-Y는 상대적으로 매우 좋은 추정량을 제공함을 살펴보았다. 특히 NGMM-Y는 확산계 수의 모수를 추정하는데 있어서 비교 대상이 된 다른 GMM들보다 우수한 결과를 주고 있는 것을 확인 할 수 있었다. 옵션의 가격 결정에 있어서 기초자산의 가격변동을 확산모형으로 가정할 때, 추세계수의 영향은 위험중립확률척도를 고려하는 과정에서 사라지는 데 비하여, 확산계수의 영향은 사라지지 않고 옵션의 가격에 중요한 영향을 미치게 된다. 이런 점을 고려하면 NGMM-Y 추정량이 상대적인 장점을 갖는 것으로 평가된다.

개선된 GMM 추정량인 NGMM-Y 추정량은 표본의 크기가 커지는 경우에 그 성능이 더 좋아지는 특성 을 보이고 있다. GMM을 포함한 확산모형의 추정법들에 대한 점근성 연구에는 표본의 크기와 함께 관 측시간 간격이 함께 개입되므로 이에 대한 추가적인 심층적 연구가 필요하다고 보인다.

\section{References}

Aït-Sahalia, Y. (1999). Transition densities for interest rate and other nonlinear diffusions, Journal of Finance, 54, 1361-1395.

Aït-Sahalia, Y. (2002). Maximum-likelihood estimation of discretely-sampled diffusions: A closed-form approximation approach, Econometrica, 70, 223-262.

Beskos, A., Papaspiliopoulos, O., Robert, G. O. and Fearnhead, P. (2006). Exact and computationally efficient likelihood-based estimation for discretely observed diffusion processes, The Journal of the Royal Statistical Society, Series B., 68, 333-383.

Chan, K. C., Karolyi, G. A., Longstaff, F. A. and Sanders, A. B. (1992). An empirical comparison of alternative models of the short-term interest rate, Journal of Finance, 47, 1209-1227.

Cox, J. (1975). Notes on option pricing I: Constant elasticity of variance diffusions. Working paper, Stanford University (reprinted in Journal of Portfolio Management, 1996, 22, 15-17).

Cox, J., Ingersoll, J. and Ross, S. (1985). A theory of the term structure of interest rates, Econometrica, 53, 385-407.

Durham, G. and Gallant, R. (2001). Numerical Techniques for Maximum Likelihood Estimation of Continuous-Time Diffusion Processes, Technical report.

Elerian, O., Chib, S. and Shephard, N. (2001). Likelihood inference for discretely observed nonlinear diffusions, Econometrika, 69, 959-993.

Eraker, B. (2001). MCMC analysis of diffusion models with application to finance, Journal of Business \& Economic Statistics, 19, 177-191. 
Hansen, L. P. (1982). Large sample properties of generalized method of moment estimators, Econometrica, 50, 1029-1054.

Heyde, C. C. (1997). Quasi-Likelihood and Its Application: A General Approach to Optimal Parameter Estimation, Springer.

Hurn, A., Jeisman, J. and Lindsay, K. (2007). Seeing the wood for the trees: A critical evaluation of methods to estimate the parameters of stochastic differential equations, Journal of Financial Econometrics, 5, 390-455.

Imbens, G. W., Spady, R. H. and Johnson, P. (1998). Information theoretic approaches to inference in moment condition models, Econometrica, 66, 339-357.

Kim, D.-G. and Lee, Y. D. (2011). Comparison study on the performances of NLL and GMM for estimating diffusion processes, The Korean Journal of Applied Statistics, 24, 1007-1020.

Kloeden, P. and Platen, E. (1999). Numerical Solution of Stochastic Differential Equations, Springer.

Lahiri, S. N. (1996). On inconsistency of estimators based on spatial data under infill asymptotics, Sankhya, Series A, 58, 403-417.

Lahiri, S. N., Lee, Y. and Cressie, N. (2002). Efficiency of least squares estimators of spatial variogram parameters, Journal of Statistical Planning and Inference, 3, 65-85.

Lee, Y. and Lahiri, S. N. (2002). Least squares variogram fitting by spatial subsampling, Journal of the Royal Statistical Society, Series B, 64, 837-854.

Lee, Y.-D. and Lee, E.-K. (2013). An approximation of the cumulant generating functions of diffusion models and the Pseudo-likelihood estimation method, Journal of the Korean Operations Research and Management Science Society, 38, 201-216.

Lee, Y., Song, S. and Lee, E. (2012). The Delta Expansion for the Transition Density of Diffusion Models, Technical Report.

Oksendal, B. (2003). Stochastic Differential Equations: An Introduction with Applications, Springer.

Pederson, A. R. (1995). A new approach to maximum likelihood estimation for stochastic differential equations based on discrete observations, Scandinavian Journal of Statistics, 22, 55-71.

Shoji, I. and Ozaki, T. (1998). Estimation for nonlinear stochastic differential equations by a local linearization method, Stochastic Analysis and Applications, 16, 733-752.

Vasicek, O. (1977). An equilibrium characterization of the term structure, Journal of Financial Economics, 5, 177-188. 


\title{
확산모형에 대한 일반화적률추정법의 개선
}

\author{
최영수 ${ }^{a} \cdot$ 이윤동 $b, 1$ \\ ${ }^{a}$ 한국외국어대학교 수학과, ${ }^{b}$ 서강대학교 경영학부
}

(2013년 8월 4일 접수, 2013년 9월 23일 수정, 2013년 9월 23일 채택)

\section{요 약}

일반화적률추정법(GMM)은 금융자료에 대한 모형모수의 추정에 자주 이용되는 방법이다. 특히 GMM은 현대금융 공학 이론의 기본을 이루는 확산모형의 추정에도 매우 자주 사용된다. 그러나 최근의 연구에서 $\mathrm{GMM}$ 은 확산모형의 모수, 특히 확산계수에 관계되는 모수의 추정에 있어서 그 성능이 좋지 못함이 지적되었다. 본 연구에서는 GMM의 이러한 단점을 개선하기 위한 대안적 방법들을 제시하고 그 통계적 성능을 시뮬레이션 연구를 통해서 비교하게 된 다. 이런 과정을 통하여 제안되고 검토된 추정방법들 중, Shoji와 Ozaki (1998)가 제안한 국소선형근사법의 결과 를 적용하여 GMM의 성능을 개선한 NGMM-Y 추정량이 매우 우수한 성질을 가지고 있음을 확인하게 된다. 특히 NGMM-Y 추정량은 확산계수에 관계된 모수의 추정에 있어서 비교대상이 된 다른 대안적 GMM 방법들에 비하여 우수한 성질을 가지고 있음을 확인하게 된다.

주요용어: 일반화적률추정법, 확산모형, 국소선형근사법.

제 1 저자의 연구는 2013 년도 한국외국어대학교 교내학술연구비의 지원에 의하여 이루어짐. 제 2 저자의 연구는 2012년도 서강대학교 교내연구비(201210023.01)의 지원에 의하여 수행된 연구임.

1 교신저자: (121-742) 서울시 마포구 신수동 1번지, 서강대학교 경영학부, 부교수.

E-mail: widylee@sogang.ac.kr 